\title{
Relation between width of the zero-bias anomaly and Kondo temperaure in transport measurements through correlated quantum dots: Effect of asymmetric coupling to the leads
}

\author{
D. Pérez Daroca, ${ }^{1,2}$ P. Roura-Bas, ${ }^{3,2}$ and A. A. Aligia ${ }^{3,4,2}$ \\ ${ }^{1}$ Gerencia de Investigación y Aplicaciones, Comisión Nacional de \\ Energía Atómica, (1650) San Martín, Buenos Aires, Argentina \\ ${ }^{2}$ Consejo Nacional de Investigaciones Científicas y Técnicas, (1025) CABA, Argentina \\ ${ }^{3}$ Centro Atómico Bariloche, Comisión Nacional de Energía Atómica, 8400 Bariloche, Argentina \\ ${ }^{4}$ Instituto Balseiro, Comisión Nacional de Energía Atómica, 8400 Bariloche, Argentina
}

\begin{abstract}
The zero-bias anomaly at low temperatures, originated by the Kondo effect when an electric current flows through a system formed by a spin- $1 / 2$ quantum dot and two metallic contacts is theoretically investigated. In particular, we compare the width of this anomaly $2 T_{\mathrm{NE}}$ with that of the Kondo resonance in the spectral density of states $2 T_{K}^{\rho}$, obtained from a Fano fit of the corresponding curves and also with the Kondo temperature, $T_{K}^{G}$, defined from the temperature evolution of the equilibrium conductance $G(T)$. In contrast to $T_{K}^{G}$ and $2 T_{K}^{\rho}$, we found that the scale $2 T_{\mathrm{NE}}$ strongly depends on the asymmetry between the couplings of the quantum dot to the leads while the total hybridization is kept constant. While the three scales are of the same order of magnitude, $2 T_{\mathrm{NE}}$ and $T_{K}^{\rho}$ agree only in the case of large asymmetry between the different tunneling couplings of the contacts and the quantum dot. On the other hand, for similar couplings, $T_{\mathrm{NE}}$ becomes larger than $T_{K}^{\rho}$, reaching the maximum deviation, of the order of $30 \%$, for identical couplings. The fact that an additional parameter to $T_{\mathrm{NE}}$ is needed to characterize the Kondo effect, weakenig the universality properties, points that some caution should be taken in the usual identification in experiments of the low temperature width of the zero-bias anomaly with the Kondo scale. Furthermore, our results indicate that the ratios $T_{\mathrm{NE}} / T_{K}^{G}$ and $T_{K}^{\rho} / T_{K}^{G}$ depend on the range used for the fitting.
\end{abstract}

PACS numbers: 73.23.-b, 71.10.Hf, 75.20.Hr

\section{INTRODUCTION}

The Kondo effect is one of the most relevant examples of the nontrivial role of correlations in quantum manybody systems $\stackrel{1.2}{=}$ Initially observed in magnetic impurities embedded in metals, is nowadays the most interesting regime and often found at low temperatures when measuring the electric current through quantum dots (QDs) in semiconducting materials,,-10 carbon nanotubes, $11-18$ and molecular systems,,$\underline{19}-28$ in which the QD acts as the magnetic impurity. In their more usual realizations, the Kondo effect can be understood as the screening of the impurity magnetic moment by the surrounding free conduction electrons forming a many-body singlet. A remarkable feature of this phenomena is given by its universality. Different physical properties depending on temperature, $T$, bias voltage, $V_{b}$, and magnetic field, $B$, among others, display an universal behavior once they are properly scaled by the Kondo temperature $T_{K}, \underline{7,8}$

The Kondo temperature is a many-body energy scale (here we take the Boltzmann $k_{B}=1$ ) that can be thought as the binding energy of the spin singlet. A precise determination of this energy is always desirable. For the simplest theoretical case in which a single interacting spin degenerate level, at energy $E_{d}$ below the Fermi one, is coupled via the hopping, $V$, to the conduction electrons there is a well defined analytical expression of this magnitude. ${ }^{2}$ The same is true for two-level or two-dot generalizations with SU(4) symmetry like QDs in carbon nanotubes,, $11-18$ or silicon nanowires $\stackrel{29}{\stackrel{9}{9}}$ or a system with two capacitively

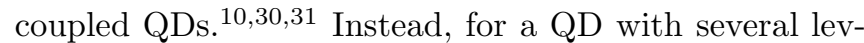
els coupled with two single-band leads, analytical expressions for $T_{K}$ are more difficult to obtain and when more than one level is occupied a scenario with several stages of the Kondo effect is the general situation ${ }^{32-34}$ In addition, the Kondo temperature scale can be obtained numerically from the low-temperature behavior of several magnitudes, like thermodynamics properties, for instance the impurity entropy $\stackrel{35,36}{5}$ or dynamical ones, like the width of the Kondo peak (the one located near the Fermi energy) in the impurity spectral density $\underline{37}$ The resulting $T_{K}$ obtained from different properties are different, although of the same order of magnitude.

In general, and particularly for the one-level singlechannel system, measurements of the electrical current through the impurity, $J$, and its derivative with respect to the bias voltage $V_{b}$, the conductance $G\left(V_{b}, T\right)=$ $d J / d V_{b}$, characterize the Kondo phenomenon. At low enough temperature, $G\left(V_{b}, T\right)$ as a function of the bias voltage has a peak at $V_{b}=0$, the zero-bias anomaly (ZBA). The peak has a width which is narrow compared to the other energy scales of the system. Increasing $T$ broadens the peak until it completely disappears. In addition, under an applied magnetic field, a splitting of ZBA appears. These properties of the ZBA represent the most clear evidence of the Kondo effect. $\frac{4}{4}$

In case of spin- $1 / 2$ QDs, the Kondo temperature is commonly extracted from i) fitting the temperaturedependence of the equilibrium conductance $G(0, T)=$ $d J /\left.d V_{b}\right|_{V_{b}=0}$, which follows a phenomenological ex- 
pression obtained using the numerical renormalization group ${ }^{3.38}$

$$
G(0, T)=\frac{G_{s}}{\left[1+\left(2^{1 / s}-1\right)\left(T / T_{K}^{G}\right)^{2}\right]^{s}},
$$

where $s=0.22$, and $G_{s}$ is the conductance at temperature $T=0$ being $T_{K}^{G}$ the only adjustable parameter and, ii) extracted from a fitting of the full width at half maximum (FWHM) of the zero-bias anomaly using the expression $^{39.40}$ (using the electron charge $|e|=1$ )

$$
\operatorname{FWHM}(T)=\sqrt{(\beta T)^{2}+\left(2 T_{K}\right)^{2}},
$$

with $\beta$ an extra fitting parameter. This expression for the FWHM gives the result $2 T_{K}$ at zero temperature. Usually, to define the FWHM, the Fano formula ${ }^{41}$ is used, which at very low temperatures directly relates the width of the ZBA with a Kondo temperature

$$
G\left(V_{b}, T=0\right)=C \frac{(\epsilon+q)^{2}}{1+\epsilon^{2}}, \quad \epsilon=\frac{V_{b}-\epsilon_{0}}{\Gamma},
$$

where $\epsilon_{0}$ is the center of the ZBA. The parameter $q$ represents the degree of asymmetry in the line shape which continuously evolves from a dip for $q=0$ to a peak for $q \rightarrow+\infty \underline{42}$. In both limiting cases, the fitting function reduces to a constant plus a Lorentzian, and $\Gamma$ is the half width of the dip or peak. Usually in experiments $\Gamma$ is identified as the Kondo scale, which we denote as $T_{\mathrm{NE}}$.

A similar Fano fit can be done for the spectral density of the impurity $\rho(\omega)$, leading to a third possible definition of the Kondo scale $T_{K}^{\rho}$.

One might argue that since the Kondo effect is an equilibrium phenomenon, which is destroyed by an application of a large bias voltage $V_{b}$, a quantity such as $T_{\mathrm{NE}}$ obtained from non-equilibrium measurements is not a good representation of the Kondo scale. However, calculations using perturbative renormalization group and poor mans scaling, valid when the largest of $e V_{b}$ and the Zeeman energy $B$ is much larger that the equilibrium Kondo scale $T_{K}$, find that $G\left(V_{b}\right)$ and the magnetization are universal functions of $e V_{b} / T_{K}$ and $B / T_{K} \stackrel{43}{\underline{a}}$ In Ref. 44, the authors use using real-time renormalization group calculations to propose a scaling function for $G\left(V_{b}\right)$ more involved than Eq, (3). Therefore in principle $T_{K}$ can be extracted from non-equilibrium measurements. We note that both works assumed symmetric coupling to the leads.

The main message of our work is that while $T_{K}^{G}$ and $T_{K}^{\rho}$ do not depend on the asymmetry of the coupling of the QD to the leads, the width of the zero-bias anomaly $2 T_{\mathrm{NE}}$ does. Therefore, part of the universality is lost, since for example the dependence of different quantities on magnetic field or temperature, do not depend only on $T_{\mathrm{NE}}$ but also on the asymmetry ratio. Then, $T_{\mathrm{NE}}$ cannot be considered as a Kondo temperature, although it is closely related to this concept. We also show that the result of the Fano fit Eq. (3) depends markedly on the range of values chosen for the fit. Both results are relevant for experiments, as discussed below.

The half width at half maximum of $\rho(\omega)$ is other frequently used definition of the Kondo temperature, but this quantity is difficult to access experimentally. Nevertheless, the spectral density has been measured in a three-terminal quantum ring, $\stackrel{45}{=}$ and a splitting of Kondo resonance for a high enough bias voltage has been observed. A problem of using the half width at half maximum is that it depends on the subtraction of a background. We discuss this point in more detail in Section IIIB Another possibility to define a Kondo scale is from the dependence of the conductance for $V_{b}, T, B \rightarrow 0$, where $B$ is the magnetic field $\stackrel{7,24,46-54}{-5 e}$ would like to mention here that while expanding Eq. (1) in powers of $T$ leads to the correct quadratic dependence of the deviation $G(T)-G(0)$, the coefficient is not correct. $\underline{54}$ Since the concept of scaling is usually used for a whole curve and not just a leading behavior, we prefer to use $T_{K}^{G}$ as the Kondo scale rather than a similar quantity derived from some leading term in the expansion of the conductance. For this reason, we restrict the discussion in our paper to the relation between $T_{\mathrm{NE}}$ and the Kondo scales $T_{K}^{G}$ and $T_{K}^{\rho}$.

In Ref. 6, W. G. van der Wiel et al., pointed out that applying a finite bias voltage introduces dephasing even at very low temperatures which leads to a possible deviation of $T_{\mathrm{NE}}$ from the values of $T_{K}^{G}$ obtained from Eq. (11). In this work, we discuss this deviation and show that there is, in addition, a geometrical mechanism that also introduces differences in the magnitudes extracted from Eqs. (11), (2) and (3). This is the asymmetry between the tunneling couplings of the QD and the leads. Previous works have studied the relation between zero-bias anomaly and Kondo temperature, for instance in Refs. 44, 55, and 56. However, the effect of asymmetry has not been discussed in detail.

We represent the QD by the spin-1/2 Anderson impurity model (AIM) and study the differential conductance by using the non-crossing approximation (NCA) in its non-equilibrium extension. To complement the results, particularly at low temperatures, we also use renormalized perturbation theory (RPT). As discussed in Section [I these approaches are complementary. We obtain that the Fano fit depends on the range of values used in the fitting procedure. This is supported by calculations using the numerical renormalization group (NRG). We also find out that $T_{\mathrm{NE}}$ varies with the asymmetry while the total coupling is set to be constant. This behavior is against the expected universality of the Kondo scale, which should roughly depend only on the energy level position and the total coupling. We find that $T_{\mathrm{NE}}$ is approximately $30 \%$ larger than $T_{K}^{\rho}$ for symmetric couplings and only tends to same value for large asymmetry.

Our results are relevant for spectroscopic measurements using a scanning tunnel microscopy (STM) per- 
formed over magnetic impurities (atoms and molecules) deposited on metallic surfaces. In these measurements the symmetry of the couplings between the magnetic impurity and the surface, and the magnetic impurity and the tip is an important issue. In particular, experiments where the tip is moved over the surface, are examples of the change in the symmetry of the aforementioned couplings. For instance, in Ref. 57 such measurements are made for a system consisting in a Co atom on $\mathrm{Cu}(111)$ and the tip of the STM is moved vertically on top of the Co atom until contact. The authors characterized the point of contact by the physical situation at which the curve $G\left(V_{b}\right)$ vs $V_{b}$ becomes symmetric, which in turn means that both couplings, $\mathrm{Co}-\mathrm{Cu}$ and Co-tip are approximately equal. .56 .58 In the experiment, the width of $G\left(V_{b}\right)$ varies due to the monotonic increase of the coupling Co-tip when the tip is moved towards the Co atom and also for the more subtle variation of the asymmetry between $\mathrm{Co}-\mathrm{Cu}$ and Co-tip couplings, which seems to have been missed in previous work. A similar experimental procedure is used for a system consisting on a Co atom adsorbed on $\mathrm{Cu}(100)$ and $\mathrm{Cu}(111)$ surfaces, as described in Refs. 5962 .

We find that $T_{\mathrm{NE}}$ deviates from $T_{K}^{\rho}$ in experimental setups for which the tunneling couplings between QD and the leads are approximately the same. Furthermore, the relations of both quantities to $T_{K}^{G}$ given by Eq. (11) depend on the range of voltages or energies used in the fitting procedure. This is important, since $T_{\mathrm{NE}}$ is widely used as an estimation of $T_{K}$ in different classes of experiments. ${ }^{59,63}-69$ For example, in Ref. 64 a "Kondo temperature" $T_{\mathrm{NE}}=92 \mathrm{~K}$ for a Co impurity on $\mathrm{Ag}(111)$ is reported, while in Ref. 69 a distribution of $T_{\mathrm{NE}}$ with an average $52.1 \pm 9.4 \mathrm{~K}$ was found for the same system. While part of the discrepancy might be due to the variation of the surface density of states $\underline{69}$ the nearly four times wider range of voltages used in Ref. 64 in the fitting procedure can explain the different $T_{\mathrm{NE}}$ as we shall show.

The paper is organized as follows. In Sec. III we describe the IAM and the method we used. In Sec. III we discuss the properties of the differential conductance and its dependence with the couplings to the leads. Sec. IV contains a summary and a discussion.

\section{MODEL AND METHODS}

As we have mentioned, we use the spin- $1 / 2$ impurity AIM to describe the molecular or semiconducting QD. This choice does not limit the qualitative validity our findings. In Section IV we address the more general case of large values of the molecular spin and also total and partial screening of it.

The model is composed by a single level characterized by an energy $E_{d}$ below the Fermi energy (which we choose at the origin of energies) coupled to two conduction leads.
The Hamiltonian reads as follows

$$
\begin{aligned}
H= & E_{d} n_{d}+U n_{d \uparrow} n_{d \downarrow}+\sum_{\nu k \sigma} \epsilon_{k}^{\nu} c_{\nu k \sigma}^{\dagger} c_{\nu k \sigma} \\
& +\sum_{\nu k \sigma}\left(V_{k}^{\nu} d_{\sigma}^{\dagger} c_{\nu k \sigma}+\text { H.c. }\right)
\end{aligned}
$$

with $n_{d}=\sum_{\sigma} n_{d \sigma}, n_{d \sigma}=d_{\sigma}^{\dagger} d_{\sigma}$. The operator $d_{\sigma}^{\dagger}$ creates an electron with spin $\sigma$ at the single level of the QD while the operators $c_{\nu k \sigma}^{\dagger}$ create conduction electrons at the leads. Depending on the specific experiment, they can represent left and right leads when conduction through a QD is studied, or the metallic substrate $(\nu=S)$ and the tip $(\nu=T)$ of the STM in scanning tunneling spectroscopy. The parameters $V_{k}^{\nu}$ describe the hopping elements between the leads and the QD. For most of our results, we take the value of the Coulomb repulsion to be infinite, $U \rightarrow \infty$ and analyze the model within the Kondo regime, $-E_{d} \gg \Delta$, being $\Delta$ the resonant level half-width. Finite values of $U$ within this regime only change the Kondo scale while the present analysis remains valid.

In the case of having different chemical potentials $\mu_{\nu}$ in the metallic contacts, a constant electric current flows through the QD in the steady state. We take the same temperature $T$ for all elements of the setup and fix the chemical potentials to be $\mu_{\nu}=-e \gamma_{\nu} V_{b} / 2$ with the sign $\gamma_{\nu}=-(+)$ for $S(T)$ being $V_{b}$ the bias voltage, as a reference.

The charge current through the QD is given by $\underline{\underline{70}}-\underline{\underline{72}}$

$$
J=\frac{2 e \pi}{h} A(\alpha) \Delta \int d \omega \rho(\omega)\left(f_{S}(\omega)-f_{T}(\omega)\right) .
$$

Here the energy $\Delta$ incorporates both, the substrate-dot and tip-dot couplings, $\Delta=\Delta_{S}+\Delta_{T}$, being $\Delta_{\nu}=$ $\pi \sum_{k}\left|V_{k}^{\nu}\right|^{2} \delta\left(\omega-\epsilon_{k}^{\nu}\right)=\pi V_{\nu}^{2} \rho_{\nu}$ assumed independent of energy. Furthermore, $\left.f_{\nu}(\omega)=1 /\left[\exp \left(\omega-\mu_{\nu} / T\right)+1\right)\right]$ is the Fermi distribution associated to the lead $\nu$, and the spectral function of the QD per spin, is denoted by $\rho(\omega)$. Regarding the factor $A(\alpha)=4 \alpha /(\alpha+1)^{2}$, it represents the asymmetry in the device geometry being $\alpha=\Delta_{S} / \Delta_{T}$ the ratio of the tunneling couplings.

For the calculation of $\rho(\omega)$ entering Eq. (5), we mainly use the non-equilibrium non-crossing approximation $(\mathrm{NCA}) \geq 1,73,74$. The non-equilibrium NCA technique is one of the standard tools for calculating the spectral density of the dot within the Kondo regime in which the population of the dot is near 1. NCA has being successfully applied to the study of a variety of systems such as two-level QD's and $\mathrm{C}_{60}$ molecules displaying a quantum phase transition, $26,37,75,76$ or a nanoscale Si transistor ${ }^{29}$ among others. Few alternatives exist out of equilibrium, like renormalized perturbation theory $53,54,72,77,78$, Fermiliquid approaches $\frac{79}{}$ and slave bosons, $\underline{80,81}$ which are restricted to small voltage and temperature, equations of motion with some difficulties to reproduce the Kondo energy scale,,$\underline{82} \underline{-84}$ or real-time renormalization group $\underline{44,55}$ Recently, a variational approach has been proposed $\stackrel{85}{8}$ 
Nevertheless, the NCA has some limitations at very low temperatures (typically below $0.05 T_{K}^{G}$ ). In particular, it does not satisfy accurately the Friedel sum rule at zero temperature 17 For this reason, we also used the approach of renormalized perturbation theory (RPT) used before by one of us. ${ }^{54,72}$ It consists of using renormalized parameters for $E_{d}, U$ and $\Delta$ obtained at $V_{b}=T=0$ by a numerical-renormalization-group calculation,,$\underline{53,86}$ and incorporating perturbations up to second order in the renormalized $U$. At equilibrium, the method provides results that coincide with state-of-the art techniques for the dependence of the conductance with magnetic field $B\left(c_{B}\right)^{53}$ and temperature $\left(c_{T}\right)^{\underline{54}}$ to second order in $B$ or $T$. An analytical expression for $c_{T}$ in terms of the renormalized parameters was provided $\underline{54}$ However, for energy scales of the order of $T_{K}^{G}$ or larger, the method loses accuracy and in particular if fails to give a splitting of the spectral density for $e V_{b}>T_{K}^{G}$, which is however well reproduced by the NCA.

\section{RESULTS}

In what follows we set $\Delta=1$ as our unity of energy, $E_{d}=-4$ for the energy level of the QD and $U \rightarrow \infty$, unless otherwise stated. Some results are presented with $E_{d}=-6$, and some RPT results in the symmetric case $U=8, E_{d}=-4$ are also shown. The choice of $E_{d}=-4$ does not affect our discussion as long as the Kondo regime $\left|E_{d}\right| \gg \Delta$. As usual, we assume a constant conduction density of states with bandwidth $2 D$. We use $D=10$.

\section{A. Nonequilibrium conductance}

We start our discussion by giving a brief description of the experiment recently made by Choi et al. in Ref. 59, in which a $\mathrm{Co}$ atom is deposited on a $\mathrm{Cu}(111)$ surface. A STM with a tip that also contains $\mathrm{Cu}$ is placed vertically over the adsorbed Co atom and is used to measure the tunneling current. From a distance tip-Co large enough, characterized by a tip-Co coupling $\Delta_{T} \ll \Delta_{S}$, the authors move the tip towards the surface till contact, which is defined by the condition of getting a symmetric curve of the conductance as a function of bias voltage $G\left(-V_{b}\right)=G\left(V_{b}\right)$.

In Fig. 11we show the differential conductance $G\left(V_{b}, T\right)$ as a function of bias voltage $V_{b}$ for several values of $\Delta_{T}$ and temperature $T$ low enough so that the conductance has already reached the saturated value for $T=0$ (in practice we have taken $T \sim T_{K}^{G} / 20$ where $T_{K}^{G}$ depends on $\left.\Delta_{T}\right)$. As expected, as $\Delta_{T}$ increases, $G$ also increases and becomes more symmetric, in qualitative agreement the results already presented in Fig. 1(c) of Ref. 59. The different curves, from bottom to top, represent the excursion of the tip as vertically approaches the adsorbed $\mathrm{Co}$ atom. We have fixed the hybridization $\mathrm{Co}-\mathrm{Cu}$ to be $\Delta_{S}=0.5$. We simulate larger distances between the Co

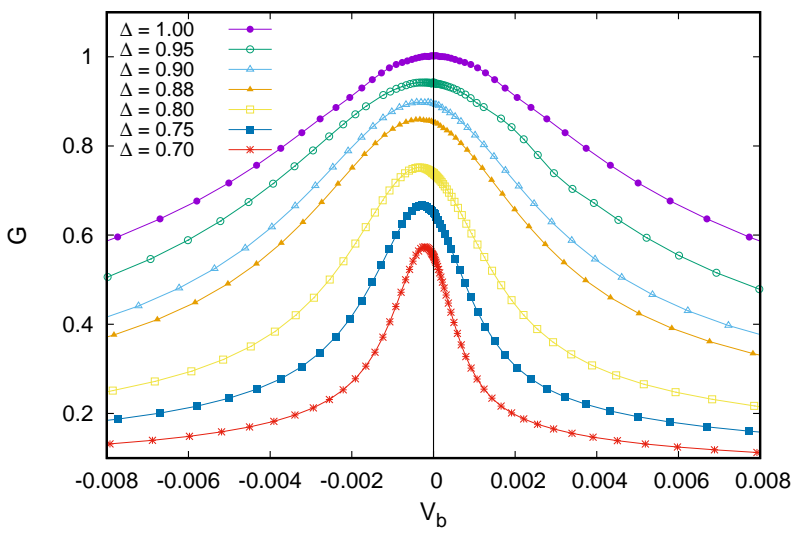

FIG. 1. (Color online) Differential conductance as a function of the applied bias voltage for $\Delta_{S}=0.5$ and several values of $\Delta_{T}$ from 0.2 (largest distance) to 0.5 (point contact).

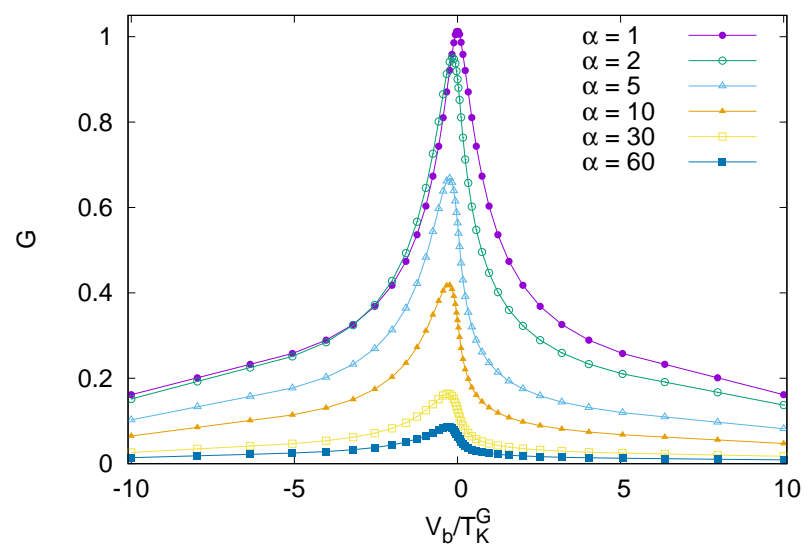

FIG. 2. (Color online) Differential conductance as a function of the applied bias voltage for $\Delta=1$ and several values of $\alpha$.

atom and the STM tip by smaller coupling $\Delta_{T}$. As soon as the distance is reduced, the value of $\Delta_{T}$ increases until the point contact is reached. We define the point contact by $\Delta_{T}=\Delta_{S}$ giving a total coupling of $\Delta=1$.

The main features of the different curves are that the width, intensity and symmetry increase as $\Delta$ does. The increase of the width is related to the increase of the Kondo scale, which in turn, depends on the total coupling $\Delta$. The increase of the intensity is due to the increase of the asymmetry factor $A(\alpha)$, which reaches $A(\alpha)=1$ for the point contact. Finally, the symmetry increases as $\Delta_{T}$ does. This is related with the fact that for $\Delta_{T} \ll$ $\Delta_{S}, G\left(V_{b}\right)$ mimics the spectral density $\rho(\omega)$ which is in turn asymmetric due to the infinite value of $U, 56,58$ while for the opposite limit, $\Delta_{T} \sim \Delta_{S}, G\left(-V_{b}\right)=\left(V_{b}\right)$ as a consequence the reflection $\mathrm{S} \leftrightarrow \mathrm{T}$ symmetry.

The increase in the width of the differential conductance as $\Delta_{T}$ increases is due to the addition of two effects that cannot be disentangled in the figure: the increase in $T_{K}^{G}$ and the decrease in the asymmetry of the couplings. In order to separate these effects, we show in Fig. 2 the results for the differential conductance for a fixed to- 


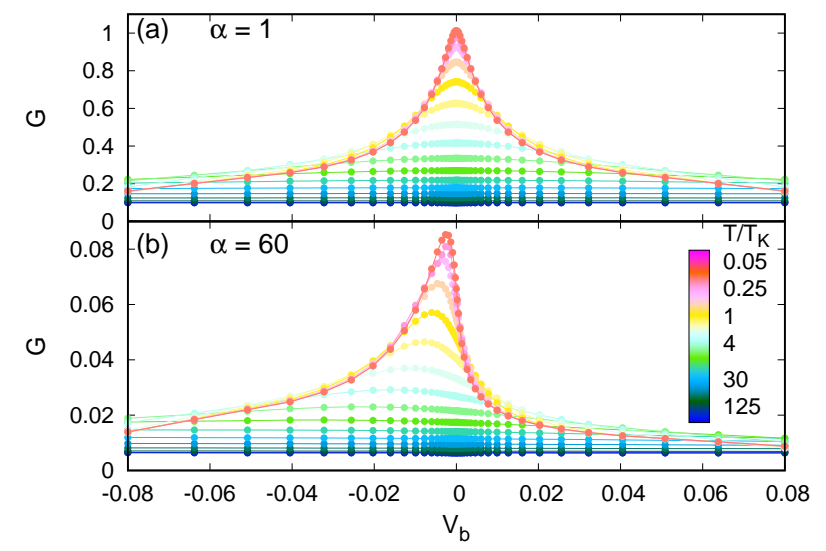

FIG. 3. (Color online) Temperature evolution of the differential conductance as a function of the applied bias voltage for two selected values of the asymmetry parameter $\alpha$. The temperature range covers the region from $125 T_{K}^{G}$ to $T_{K}^{G} / 20$.

tal coupling $\Delta=1$ as a function of the applied voltage and the asymmetry ratio $\alpha=\Delta_{S} / \Delta_{T}$. In this way, the results become independent of $\Delta$ but retain the dependence in the asymmetry, which is the main ingredient in the present discussion. As in Fig. 1, the symmetry and intensity increase as $\alpha$ is reduced. However, it is expected that the Kondo temperature remains constant simply because it depends on the total coupling $\Delta$ and not on the asymmetry ratio $\alpha=\Delta_{S} / \Delta_{T}$. From the spectral density, the Kondo temperature is given by the half-width al half-maximum of the Kondo peak. It is well known that the shape of the Kondo resonance in the spectral density does not depend on the asymmetry coupling, see for instance Fig. 1 in Ref. 58. We have verified that this is actually the case for the whole values of $\alpha$ from $\alpha=1$ to $\alpha=60$. Therefore, the width of this resonance, or that obtained from the Fano fit, which we denote as $2 T_{K}^{\rho}$, is independent of $\alpha$. However from the figure, particularly for small values of $\alpha$, it is clear that the width of the peak in the differential conductance $G\left(V_{b}\right)\left(2 T_{\mathrm{NE}}\right.$ from a Fano fit) narrows as $\alpha$ increases.

The temperature evolution of the differential conductance can also be used to determine the Kondo scale $T_{K}^{G}$ by using Eq. (11). In Fig. 3 we show the temperature dependence of $G\left(V_{b}\right)$ for the symmetric case $\alpha=1$ (top panel), which can be related with the point contact regime of the experiment in Ref. 59, and for the opposite strong asymmetric one $\alpha=60$. From the figure, it is not obvious that a fitting of the values of $G\left(V_{b}=0\right)$ (obtained from the non-equilibrium calculation) with Eq. (11) gives the same result of $T_{K}^{G}$ for both cases. However in Fig. 4 we confirm that this is actually the case. We have verified that the same value of $T_{K}^{G}=0.00797$ is obtained independently of the value of $\alpha$. Fig. 4 shows the temperature dependence of the equilibrium conductance for the two selected values of the asymmetry parameter, $\alpha=1$ and $\alpha=60$. Calculating $G(T)$ at $V_{b}=0$ from an equilibrium calculation gives the same result.

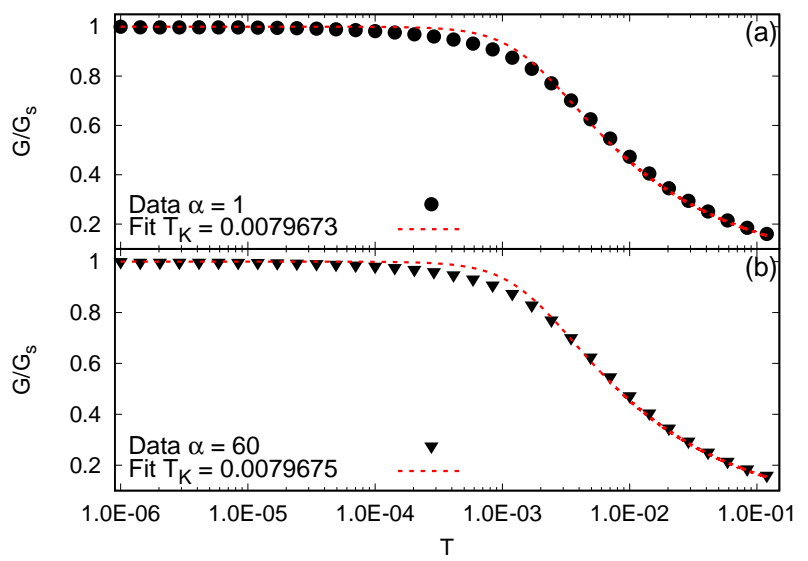

FIG. 4. (Color online) Temperature evolution of the equilibrium conductance (discrete points) normalized by its saturation values and the corresponding fitting with Eq1 (full red lines).

\section{B. Fitting procedure to determine the widths of the curves}

For a quantitative analysis of the effect of the asymmetry in the widths of the Kondo resonances in the differential conductance $G\left(V_{b}\right)$ and the spectral density $\rho(\omega)$ at small temperature, we need some procedure to determine these widths. Experimentally Fano fits described by Eq. (3) after subtracting a background, are the most widely used. The Frota function ${ }^{87}$ is also used but it does not permit asymmetric shapes and therefore it is not useful for our purpose. Theoretically, the half-width at half maximum of the corresponding curve is also used. However, we have found that this leads to an overestimation of the widths due to the fact that the Kondo peak is mounted on the tails of the charge transfer peaks. These peaks in the spectral density are centered at energies $E_{d}$ and $E_{d}+U$ and have total width near $4 \Delta, 56,88$ Therefore, we analyze the widths using the Fano formula rewritten in the following form using $q=1 / x$ in Eq.(3) and adding a constant background $A$ :

$$
G=A+B \frac{(1+x \epsilon)^{2}}{1+\epsilon^{2}}
$$

Note that for the symmetric case $\alpha=1$, which corresponds to $x=0, G$ is a constant plus a Lorentzian function with half-width $\Gamma$. Examples of fits of $G\left(V_{b}\right)$ are given in Fig. [5]

We identify the value of $\Gamma$ that results from the fit of $G\left(V_{b}\right)$ at small enough temperatures with $T_{\mathrm{NE}}$. Similarly, the value of $\Gamma$ obtained fitting $\rho(\omega)$ gives $T_{K}^{\rho}$. While the result of the fit is unambiguous, a difficulty of this procedure (also found experimentally ${ }^{89}$ ) is that the resulting $\Gamma$ depends on the window (range of values of the abscissa) of the fit. In some experimental work with scan-

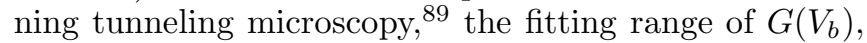




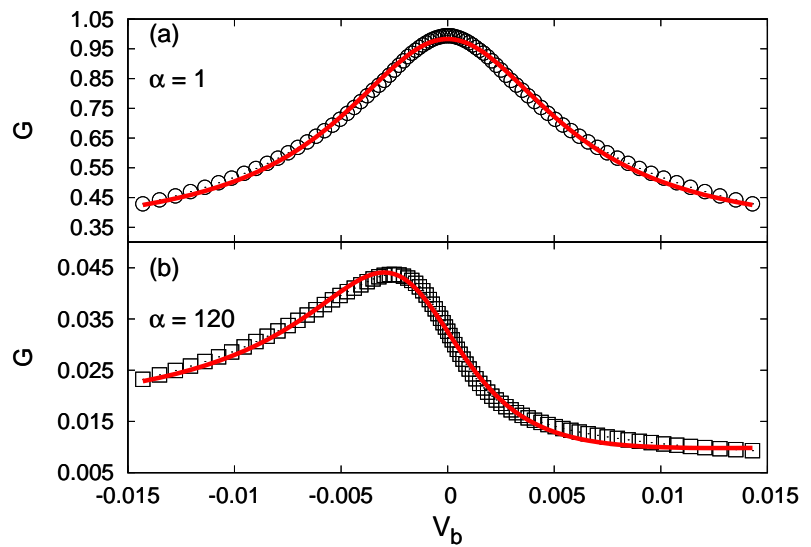

FIG. 5. (Color online) Differential conductance (discrete points) as a function of the bias voltage for $\alpha=1$ (top panel) and $\alpha=120$ (bottom panel). The continuous line corresponds to the Fano fitting with Eq. (6).

$-W \leq e V_{b} \leq W$ was established as $W=1.5 T_{\mathrm{NE}}$ obtaining $T_{\mathrm{NE}}$ from the fit and then changing $W$ if it does not coincide with $1.5 T_{\mathrm{NE}}$ until convergence. In our case, it is simpler to define the range $W$ in terms of the Kondo temperature determined from the conductance at equilibrium $T_{K}^{G}$, which is defined unambiguously.

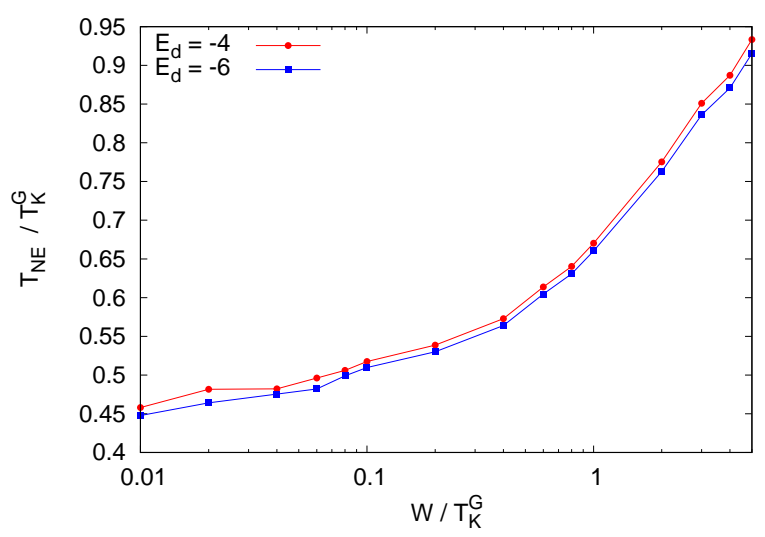

FIG. 6. (Color online) $T_{\mathrm{NE}}$ obtained from the Fano fit of the differential conductance as a function of the window of the fit $-W \leq e V_{b} \leq W$ for $\alpha=1$ and two values of $E_{d}$.

In Fig. 6] we show the half-width $\Gamma$ of the ZBA in the differential conductance obtained from the fit Eq. (6) for different fitting windows measured in units of $T_{K}^{G}$. Several conclusions can be drawn from this figure. In spite of taking the symmetric case $\alpha=1$, for which $x=0$, the shape of the curve (corresponding to the top panel in Fig. (5) is not Lorentzian. Otherwise its width would be independent of the window of the fit. However, normalizing the width with $T_{K}^{G}$ its shape is universal. It is the same for different values of $E_{d}$. The same is true for the values of $A$ and $B$ of the fit using Eq. (6) (not shown). The fit for $W \ll T_{K}^{G}$ becomes meaningless since $A$ tends to the quantum of conductance $G_{0}=2 e^{2} / h$ and $B$ becomes very small. For $W \gg T_{K}^{G}$ the fit has too much weight on the tails of the Kondo resonance. The choice $W \sim 1.5 T_{\mathrm{NE}}$ made by experimentalists $\frac{89}{}$ seems reasonable. This corresponds approximately to $W=2 T_{K}^{G}$. The latter choice allows us to avoid a self-consistent procedure to determine $W$.

Motivated by the arguments above, we take $W=2 T_{K}^{G}$ for all the calculations of $T_{\mathrm{NE}}$ and $T_{K}^{\rho}$ that follow, except in the discussion of Fig. 7 of the next paragraph.

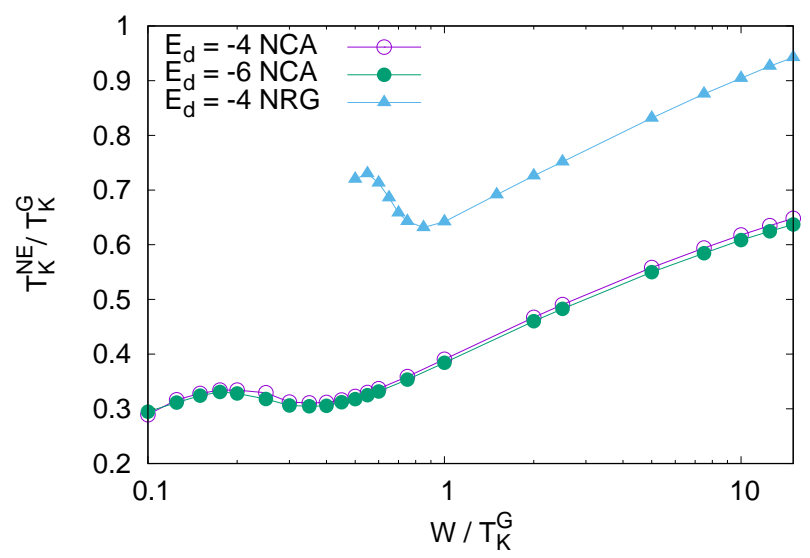

FIG. 7. (Color online) $T_{K}^{\rho}$ obtained from the Fano fit of the spectral density as a function of the window of the fit $-W \leq$ $e V_{b} \leq W$ for two values of $E_{d}$ and two techniques.

Qualitatively, the same general features are shared in the fits of the spectral density $\rho(\omega)$, as it is shown in Fig. 7. We also show in the figure the results obtained using the numerical renormalization group (NRG) $\stackrel{90}{?}$ In spite of the known different shape between the spectral densities calculated by $\mathrm{NCA}$ and $\mathrm{NRG}, 91$ one can see that qualitatively the same trend of increasing $T_{K}^{\rho}$ with increasing $W$ for $W \sim T_{K}^{G}$ takes place for both approaches. The difference is that the ratio $T_{K}^{\rho} / T_{K}^{G}$ is about 1.5 times greater with NRG.

\section{Comparison of the width of the zero-bias anomaly with the Kondo scale}

In Fig. 8 we show the half width $T_{\mathrm{NE}}$ obtained from the fit of the differential conductance using the procedure described in Section IIIB as a function of the asymmetry parameter $\alpha$ for two techniques, NCA and RPT. We discuss first the NCA results. We remind the reader that for NCA, $T_{K}^{G}=0.00797$ independent of $\alpha$. The results allows us to quantify the narrowing of $G\left(V_{b}\right)$ with increasing $\alpha$ already apparent in Fig. 2. The behavior of $T_{\mathrm{NE}}$ for moderate and small values of $\alpha$ is unexpected, and missed in previous studies $\stackrel{57}{\underline{5 n}}$ Ine bottom panel of the figure we show the ratio of the widths derived from the fits of the differential conductance $G\left(V_{b}\right)$ and the spectral density $\rho(\omega)$. As expected, this ratio tends to 1 for large $\alpha$ and small temperatures in the Kondo limit. For large 


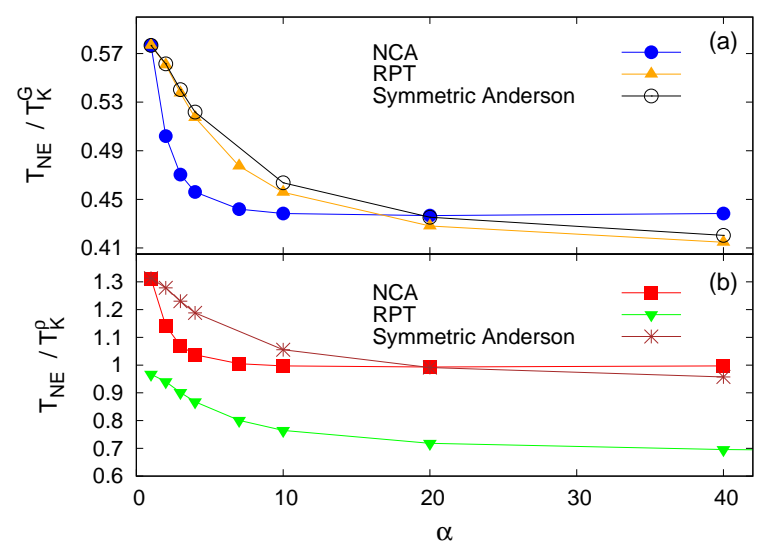

FIG. 8. (Color online) Ratio of $T_{\mathrm{NE}}$ obtained from the Fano fit [ $\Gamma$ in Eq. (6)] $G\left(V_{b}\right)$ to $T_{K}^{G}$ (top panel) and ratio $T_{\mathrm{NE}} / T_{K}^{\rho}$ (bottom panel) as a function of $\alpha$ for $T=0.001 T_{K}^{G}$. Triangles are RPT results (normalized to the same value for $\alpha=1$ in the top panel).

asymmetry the dot is practically at equilibrium with the substrate, so that there is a Kondo peak near the chemical potential of the substrate $\mu_{S}=e V_{b} / 2$, and $G\left(V_{b}\right)$ mimics the spectral density $G\left(V_{b}\right) \sim \frac{e^{2}}{h} \pi \Delta A(\alpha) \rho\left(\mu_{T}\right)$ with $\mu_{T}=-e V_{b} / 2$ the chemical potential of the tip $\underline{58}$ Instead, in the case of symmetric couplings, like the case of point contact in the experiment of Ref. 59, we obtain that the width of the differential conductance at low temperatures $2 T_{\mathrm{NE}}$ is nearly $30 \%$ larger than the width $2 T_{K}^{\rho}$ of the spectral density of states. This agrees with previous estimates based on the half-width at half maximum of the corresponding curves ${ }^{56}$

For RPT, we have obtained $T_{K}^{G}$ by a fit with Eq. (11) in the range $0<T / \widetilde{\Delta}<0.1$, where $\widetilde{\Delta}$ is the renormalized value of $\Delta . \underline{54}$. For larger temperatures the RPT results lie above the universal curve and are not quantitatively reliable. We have used here two sets of parameters: $E_{d}=-4 \Delta$ and $U \rightarrow \infty$ as in the NCA calculation [the corresponding RPT parameters are $\widetilde{\Delta}=0.00579$, $\widetilde{E}_{d} / \widetilde{\Delta}=0.161$ and $\widetilde{U} /(\pi \widetilde{\Delta})=1.025$ (Ref. 54)] , and $E_{d}=-4 \Delta, U=8$ corresponding to the symmetric Anderson model [with renormalized parameters $\widetilde{\Delta}=0.120$, $\widetilde{E}_{d} / \widetilde{\Delta}=0$ and $\widetilde{U} /(\pi \widetilde{\Delta})=0.985$ (Ref. 54 )]. From the fitting procedure we obtain $T_{K}^{G}=0.7612 \widetilde{\Delta}=0.00441$ for $U \rightarrow \infty$ and $T_{K}^{G}=0.7390 \widetilde{\Delta}=0.0887$ for $U=8$. By comparison the Bethe ansatz expression of the Kondo temperature for this case (see for example Eq. (8) of Ref. 84 ) gives $T_{K}=0.105$. The dependence with $\alpha$ is qualitatively similar with that obtained by NCA, particularly for $U=8$. However, for $U \rightarrow \infty$, the ratio $T_{\mathrm{NE}} / T_{K}^{\rho}$ is smaller and in particular it is smaller than 1 in the limit of large $\alpha$ which indicates a failure of RPT. In the symmetric case $U=-2 E_{d}$, the real part of the renormalized retarded self-energy vanishes by symmetry, while for any parameters, the renormalized lesser and greater self-energies

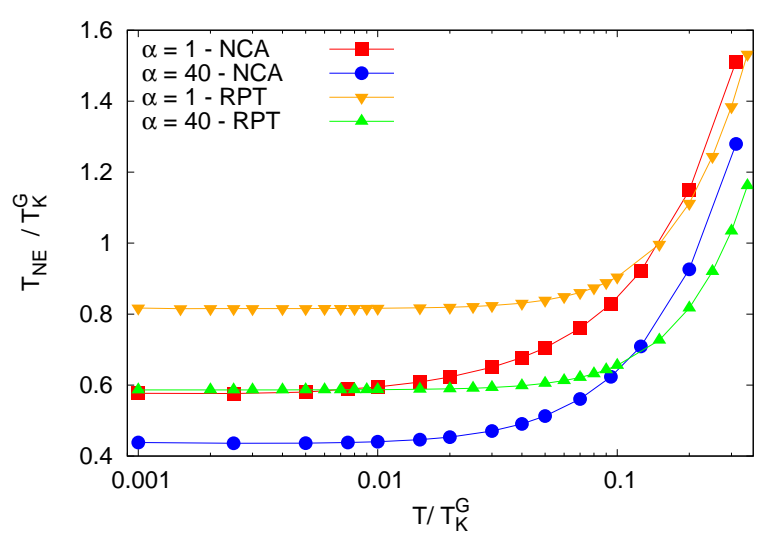

FIG. 9. (Color online) Ratio $T_{\mathrm{NE}} / T_{K}^{G}$ as a function of temperature for two values of $\alpha$ and two techniques.

and the imaginary part of the renormalized retarded selfenergy are exact to total second order in $\omega, T$ and $V_{b} \stackrel{72}{\underline{t}}$ Therefore one expects that RPT is more accurate in the symmetric case. The symmetric point is important because often experiments on quantum dots are tuned to this point at which the spectral density gets its maximum value $1 /(\pi \Delta)$ according to the Friedel sum rule, $\stackrel{72}{,}$ leading at $V_{b}=T=0$ to $G=d I / d V=(2 e / h) A(\alpha)$ in Eq. (5). From here, the asymmetry factor $A(\alpha)$ can be deduced.

In Fig. 9 we show the change with temperature of the half with of the differential conductance $T_{\mathrm{NE}}$ as a function of voltage obtained from the Fano fit Eq. (6) for NCA and RPT for $U \rightarrow \infty$. There is a strong temperature dependence. $T_{\mathrm{NE}}$ increases by a factor near 3 when the temperature reaches values of the order of $T_{K}^{G}$. This should be taken into account when Fano fits are performed on experimental data at finite temperatures. As expected from Fermi liquid theories $, 53,54,72,77-79$ the dependence resulting form RPT is quadratic for small temperature. A fit for the data for $T / T_{K}^{G}<0.1$ gives $T_{\mathrm{NE}}(T) / T_{\mathrm{NE}}(0)=1+11.0\left(T / T_{K}^{G}\right)^{2}$ for $\alpha=1$ and $T_{\mathrm{NE}}(T) / T_{\mathrm{NE}}(0)=1+12.0\left(T / T_{K}^{G}\right)^{2}$ for $\alpha=40$. In contrast, the NCA results in the same temperature range display a dependence more similar to a linear one, which is likely to be related with the shortcomings of the NCA at low temperatures.

For comparison, in Fig. 10 we show the temperature dependence of the half width of the spectral density. As expected, it is weaker than that of the differential conductance, because in the latter the effects of broadening of the spectral density and the Fermi functions in Eq. (5) are added. Here the low temperature dependence predicted by RPT for $T / T_{K}^{G}<0.1$ is $T_{K}^{\rho}(T) / T_{K}^{\rho}(0)=1+5.75\left(T / T_{K}^{G}\right)^{2}$. 


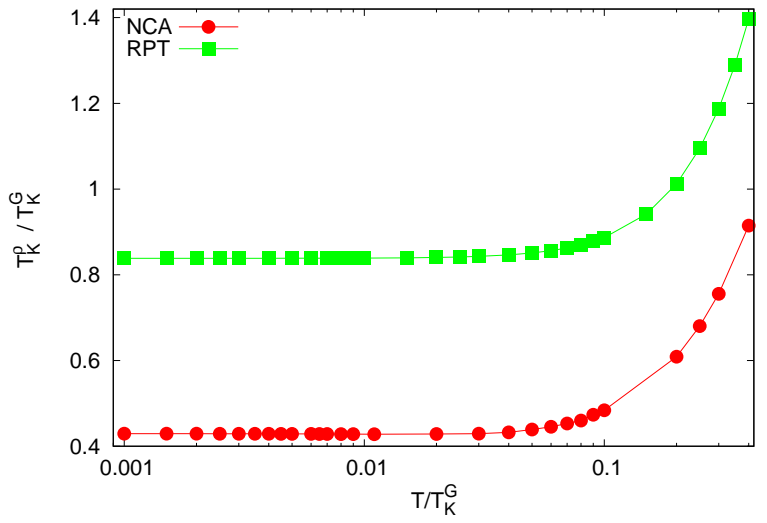

FIG. 10. (Color online) Ratio $T_{K}^{\rho} / T_{K}^{G}$ as a function of temperature for any $\alpha$ and two techniques.

\section{CONCLUSIONS}

In summary, we have investigated theoretically the width of the zero-bias anomaly, originated by the Kondo effect, when a charge current flows through a system composed by a spin- $1 / 2$ quantum dot and two metallic contacts. In particular, we have compared the energy scale represented by this width $T_{\mathrm{NE}}$ with definitions of the Kondo temperature obtained from the width of the Kondo resonance in the spectral density at equilibrium $\left(T_{K}^{\rho}\right)$ and from the well established temperature evolution of the equilibrium conductance $G(0, T)\left(T_{K}^{G}\right)$. Our results at low enough temperatures show that $T_{\mathrm{NE}}=T_{K}^{\rho}$ only in cases of large asymmetry between the different tunneling couplings of the contacts with the quantum dot. On the other hand, if the couplings tend to be similar, $T_{\mathrm{NE}}$ becomes larger than $T_{K}^{\rho}$.

The ratio $T_{\mathrm{NE}} / T_{K}^{\rho}$ reaches values as high as 1.30. Following usual experimental procedures, we have determined the above mentioned widths using a Fano fit of the line shape. Our results using NCA and also NRG show that the result depends on the range of values used in the fit. The temperature dependence of these widths is strong and stronger for the nonequilibrium conductance $G=d I / d V_{b}$ vs $V_{b}$ than for the spectral density.

As explained in the introduction, our findings are relevant for a wide range of different experiments. The asymmetry ratio between the tunnel couplings is directly related with the intensity of the differential conductance at zero bias from Eq. (5). In particular, in the Kondo limit at zero temperature $G(0,0)=2 A(\alpha) e^{2} / h$. The effects of this asymmetry in the width of the zero-bias anomaly has been missed previously and is particularly relevant in experiments in which the Kondo temperature and the asymmetry ratio are simultaneously changed. On the other hand different windows used in Fano fit can explain conflicting reports on the width of the zero-bias anomaly for the same system.

As message to experimentalists, if the precise value of the Kondo temperature matters, it is more convenient to extract it from the temperature dependence of the zerobias conductance $(G(0, T))$ than from the shape of the zero-bias anomaly as a function of bias voltage $\left(G\left(V_{b}, 0\right)\right)$. If a fit of the latter is done, the width depends on the range of the fitting (which should therefore be specified) and the asymmetry ratio.

While most of our results were calculated assuming infinite on-site repulsion $U \rightarrow \infty$. calculations in the symmetric case $U=-2 E_{d}$ and Kondo limit $-2 E_{d} \gg \Delta$ using renormalized perturbation theory confirm the main conclusions. We have also assumed a constant density of conduction electrons $\rho_{\nu}(\omega)$ around the Fermi energy for both leads. Recent experiments obtain an approximate linear dependence of $\Delta$ with the applied gate voltage indicating a variation of the density of states $\frac{92}{2}$ We expect that in this case the main result that the ratio $T_{\mathrm{NE}} / T_{K}^{\rho}$ varies from near 1.30 for symmetric coupling to 1 for very asymmetric coupling remains. We also expect that if the variation of $\rho_{\nu}(\omega)$ around the Fermi level is small on the scale of $T_{K}$, the results would be very similar as taking the average density in this scale. As an example, a step of magnitude $\Delta / 2$ in $\Delta$ [which simulates the onset of the surface band of noble metals at the (111) surface] at positions $\omega= \pm \Delta / 2$ with $\Delta \gg T_{K}$ changes $T_{K}$ by nearly three orders of magnitude, but the shape of the Kondo peak in the spectral density rescaled with $T_{K}$ is very similar, indicating that the this peak is sensitive to the value of $\rho_{\nu}(\omega)$ near the Fermi energy and not to its structure for $|\omega|>T_{K}$ (see Figs. 6 and 7 in Ref. 93).

Our results can be extended to other systems. For instance, in cases of impurity spin $S>1 / 2$, partially screened by one channel of conduction electrons, there is a similar temperature dependence of the conductance $G(0, T)$ as that given by Eq. (1) with the difference of having other values of the parameter $s$. A table of this parameter for several values of the impurity spin is given in Ref. 25. On the other hand, in case of a total compensated spin $S=1$ with two conduction channels, the corresponding expression is given in Ref. 94. It would be interesting to study the ratios of the different Kondo scales in these cases.

Acknowledgment. We are indebted to Germán Blesio for the NRG calculations presented in Fig. 7 A. A. A, was sponsored by PIP 112-201501-00506 of CONICET and PICT 2013-1045 of the ANPCyT.
1 Resistance Minimum in Dilute Magnetic Alloys, J. Kondo, Prog. Theor. Phys. 32, 37 (1964).
2 A. C. Hewson, The Kondo Problem to Heavy Fermions (Cambridge University Press, Cambridge, England, 1997), 
ISBN 9780521599474.

3 Kondo effect in a single-electron transistor, D. GoldhaberGordon, H. Shtrikman, D. Mahalu, D. Abusch-Magder, U. Meirav, and M. A. Kastner, Nature 391, 156 (1998).

4 A Tunable Kondo Effect in Quantum Dots, S. M. Cronenwett, T. H. Oosterkamp, and L. P. Kouwenhoven, Science 281, 540 (1998).

5 From the Kondo Regime to the Mixed-Valence Regime in a Single-Electron Transistor, D. Goldhaber-Gordon, J. Göres, M. A. Kastner, H. Shtrikman, D. Mahalu, and U. Meirav, Phys. Rev. Lett. 81, 5225 (1998).

6 The Kondo Effect in the Unitary Limit, W.G. van der Wiel, S. de Franceschi, T. Fujisawa, J.M. Elzerman, S. Tarucha, and L.P. Kowenhoven, Science 289, 2105 (2000).

7 Universal Scaling in Nonequilibrium Transport through a Single Channel Kondo Dot, M. Grobis, I. G. Rau, R. M. Potok, H. Shtrikman, and D. Goldhaber-Gordon, Phys. Rev. Lett. 100, 246601 (2008).

8 Spin-1/2 Kondo effect in an InAs nanowire quantum dot: Unitary limit, conductance scaling, and Zeeman splitting, A. V. Kretinin, H. Shtrikman, D. Goldhaber-Gordon, M. Hanl, A. Weichselbaum, J. von Delft, T. Costi, and D. Mahalu, Phys. Rev. B 84, 245316 (2011).

9 Pseudospin-Resolved Transport Spectroscopy of the Kondo Effect in a Double Quantum Dot, S. Amasha, A. J. Keller, I. G. Rau, A. Carmi, J. A. Katine, H. Shtrikman, Y. Oreg, and D. Goldhaber-Gordon, Phys. Rev. Lett. 110, 046604 (2013).

10 Emergent SU(4) Kondo physics in a spincharge-entangled double quantum dot, A. J. Keller, S. Amasha, I. Weymann, C. P. Moca, I. G. Rau, J. A. Katine, H. Shtrikman, G. Zaránd, and D. Goldhaber-Gordon, Nat. Phys. 10, 145 (2014).

11 Orbital Kondo effect in carbon nanotubes, P. JarilloHerrero, J. Kong, H. S. J. van der Zant, C. Dekker, L. P. Kouwenhoven, and S. De Franceschi, Nature 434, 484 (2005).

12 SU(4) Kondo Effect in Carbon Nanotubes, M.-S. Choi, R. López, and R. Aguado, Phys. Rev. Lett. 95, 067204 (2005).

13 Kondo effects in carbon nanotubes: From $S U(4)$ to $S U(2)$ symmetry, J. S. Lim, M.-S. Choi, M. Y. Choi, R. López, and R. Aguado, Phys. Rev. B 74, 205119 (2006).

14 Zero-Bias Conductance in Carbon Nanotube Quantum Dots, F. B. Anders, D. E. Logan, M. R. Galpin, and G. Finkelstein, Phys. Rev. Lett. 100, 086809 (2008).

15 Spin-polarized current and shot noise in a carbon nanotube quantum dot in the Kondo regime, S. Lipinski and D. Krychowski, Phys. Rev. B 81, 115327 (2010).

16 Transport in carbon nanotubes: Two-level SU(2) regime reveals subtle competition between Kondo and intermediate valence states, C. A. Büsser, E. Vernek, P. Orellana, G. A. Lara, E. H. Kim, A. E. Feiguin, E. V. Anda, and G. B. Martins, Phys. Rev. B 83, 125404 (2011).

17 Transition between $S U(4)$ and $S U(2)$ Kondo effect, L. Tosi, P. Roura-Bas, and A. A. Aligia, Physica B 407, 3259 (2012).

18 Magnetic-Field Dependence of Tunnel Couplings in Carbon Nanotube Quantum Dots K. Grove-Rasmussen, S. Grap, J. Paaske, K. Flensberg, S. Andergassen, V. Meden, H. I. Jorgensen, K. Muraki, and T. Fujisawa, Phys. Rev. Lett. 108, 176802 (2012).

19 Kondo resonance in a single-molecule transistor, W. Liang, M. P. Shores, M. Bockrath, J. R. Long, and H. Park, Nature 417,725 (2002).
20 Kondo Resonances and Anomalous Gate Dependence in the Electrical Conductivity of Single-Molecule Transistors, L. H. Yu, Z. K. Keane, J. W. Ciszek, L. Cheng, J. M. Tour, T. Baruah, M. R. Pederson, and D. Natelson, Phys. Rev. Lett. 95, 256803 (2005).

21 Berry-Phase Oscillations of the Kondo Effect in SingleMolecule Magnets, M. N. Leuenberger and E. R. Mucciolo, Phys. Rev. Lett. 97, 126601 (2006).

22 Tuning the Kondo Effect with a Mechanically Controllable Break Junction, J. J. Parks, A. R. Champagne, G. R. Hutchison, S. Flores-Torres, H. D. Abruña, and D. C. Ralph, Phys. Rev. Lett. 99, 026601 (2007).

23 Quantum phase transition in a single-molecule quantum dot, N. Roch, S. Florens, V. Bouchiat, W. Wernsdorfer, and F. Balestro, Nature 453, 633 (2008).

24 Universal scaling of nonequilibrium transport in the Kondo regime of single molecule devices, G. D. Scott, Z. K. Keane, J. W. Ciszek, J. M. Tour, and D. Natelson, Phys. Rev. B 79, 165413 (2009).

25 Mechanical Control of Spin States in Spin-1 Molecules and the Underscreened Kondo Effect, J. J. Parks, A. R. Champagne, T. A. Costi, W. W. Shum, A. N. Pasupathy, E. Neuscamman, S. Flores-Torres, P. S. Cornaglia, A. A. Aligia, C. A. Balseiro, G. K.-L. Chan, H. D. Abruñ a, and D. C. Ralph, Science 328, 1370 (2010).

26 Universal transport signatures in two-electron molecular quantum dots: gate-tunable Hund's rule, underscreened Kondo effect and quantum phase transitions, S. Florens, A, Freyn, N. Roch, W. Wernsdorfer, F. Balestro, P. RouraBas and A. A. Aligia, J. Phys. Condens. Matter 23, 243202 (2011); references therein.

27 Electronic read-out of a single nuclear spin using a molecular spin transistor, R. Vincent, S. Klyatskaya, M. Ruben, W. Wernsdorfer, and F. Balestro, Nature (London) 488, 357 (2012).

28 Conductance saturation in a series of highly transmitting molecular junctions T. Yelin, R. Korytár, N. Sukenik, R. Vardimon,B. Kumar, C. Nuckolls, F. Evers, and O. Tal, Nat. Mater. 15, 444 (2016).

29 Magnetic-Field Probing of an SU(4) Kondo Resonance in a Single-Atom Transistor, G. C. Tettamanzi, J. Verduijn, G. P. Lansbergen, M. Blaauboer, M. J. Calderón, R. Aguado, and S. Rogge, Phys. Rev. Lett. 108, 046803 (2012).

30 Restoring the SU(4) Kondo regime in a double quantum dot system, L. Tosi, P. Roura-Bas, and A. A. Aligia, J. Phys.: Condens. Matter 27, 335601 (2015).

31 Conditions for observing emergent SU(4) symmetry in a double quantum dot, Y. Nishikawa, O. J. Curtin, A. C. Hewson, D. J. G. Crow, and J. Bauer, Phys. Rev. B 93, 235115 (2016).

32 Kondo Effect in Real Quantum Dots, M. Pustilnik and L. I. Glazman, Phys. Rev. Lett. 87, 216601 (2001).

33 Interplay between quantum interference and Kondo effects in nonequilibrium transport through nanoscopic systems, $\mathrm{P}$. Roura-Bas, L. Tosi, A. A. Aligia, and K. Hallberg, Phys. Rev. B 84, 073406 (2011).

34 Two-color Fermi-liquid theory for transport through a multilevel Kondo impurity, D. B. Karki, C. Mora, J. von Delft, and M. N. Kiselev, Phys. Rev. B 97, 195403 (2018).

35 Non-Fermi liquid behaviour in transport through Co-doped Au chains; S. Di Napoli, A. Weichselbaum, P. Roura-Bas, A. A. Aligia, Y. Mokrousov and S. Blügel; Phys. Rev. Lett. 110, 196402 (2013).

36 Non-Fermi liquid behaviour in non-equilibrium transport 
through Co doped Au chains connected to four-fold symmetric leads; S. Di Napoli, A. Weichselbaum, P. RouraBas, A. A. Aligia; Phys. Rev. B 90, 125149 (2014).

37 Orbital Kondo spectroscopy in a double quantum dot system; L. Tosi, P. Roura-Bas and A. A. Aligia; Phys. Rev. B 88, 235427 (2013).

38 Kondo Effect in a Magnetic Field and the Magnetoresistivity of Kondo Alloys, T. A. Costi, Phys. Rev. Lett. 85, 1504 (2000).

39 Evolution of Kondo resonance from a single impurity molecule to the two-dimensional lattice, N. Tsukahara, S. Shiraki, S. Itou, N. Ohta, N. Takagi, and M. Kawai, Phys. Rev. Lett. 106, 187201 (2011).

40 Long-range ferrimagnetic order in a two-dimensional supramolecular Kondo lattice, J. Girovsky et al., Nat. Commun. 8, 15388 (2017).

41 Effects of configuration interaction on intensities and phase shifts; U. Fano, Phys. Rev. 124, 1866 (1961).

42 Theory of the Fano Resonance in the STM Tunneling Density of States due to a Single Kondo Impurity, O. Újsághy, J. Kroha, L. Szunyogh, and A. Zawadowski, Phys. Rev. Lett. 85, 2557 (2000); Spin-polarized STM for a Kondo adatom, A. C. Seridonio, F. M. Souza, and I A Shelykh, J. Phys.: Condens. Matter 21, 095003 (2009); Long-range Kondo signature of a single magnetic impurity, H. Prüser, M. Wenderoth, P. E. Dargel, A. Weismann, R. Peters, T. Pruschke, and R. G. Ulbrich, Nature Phys 7, 203 (2011); Kondo resonance lineshape of magnetic adatoms on decoupling layers, R. Žitko Phys. Rev. B 84, 195116 (2011).

43 Nonequilibrium Transport through a Kondo Dot in a Magnetic Field: Perturbation Theory and Poor Mans Scaling, A. Rosch, J. Paaske, J. Kroha, and P. Wölfle, Phys. Rev. Lett. 90, 076804 (2003).

44 Nonequilibrium Kondo model: Crossover from weak to strong coupling, M. Pletyukhov and H. Schoeller, Phys. Rev. Lett. 108, 260601 (2012).

45 Probing the Kondo Density of States in a Three-Terminal Quantum Ring, R. Leturcq, L. Schmid, K. Ensslin, Y. Meir, D.C. Driscoll, and A.C. Gossard, Phys. Rev. Lett. 95, 126603 (2005).

46 Fermi Liquid Theory for the Nonequilibrium Kondo Effect at Low Bias Voltages, A. Oguri, J. Phys. Soc. Jpn. 74, 110 (2005).

47 Universal scaling in nonequilibrium transport through an Anderson impurity J. Rincón, A. A. Aligia, and K. Hallberg, Phys. Rev. B 79, 121301(R) (2009); Phys. Rev. B 80, 079902(E) (2009); Phys. Rev. B 81, 039901(E) (2010).

$481 / N$ expansion of the nonequilibrium infinite- $U$ Anderson model, Z. Ratiani and A. Mitra, Phys. Rev. B 79, 245111 (2009).

49 Nonequilibrium conductance of asymmetric nanodevices in the Kondo regime, E. Sela and J. Malecki, Phys. Rev. B 80, 233103 (2009).

50 Universal scaling in transport out of equilibrium through a single quantum dot using the noncrossing approximation, P. Roura-Bas, Phys. Rev. B 81, 155327 (2010).

51 Nonequilibrium conductance of a nanodevice for small bias voltage, A. A. Aligia, J. Phys. Condens. Matter 24, 015306 (2012); Corrigendum 29, 069501 (2017).

52 Universal Out-of-Equilibrium Transport in KondoCorrelated Quantum Dots: Renormalized Dual Fermions on the Keldysh Contour E. Muñoz, C. J. Bolech, and S. Kirchner, Phys. Rev. Lett. 110, 016601 (2013); A. A. Aligia, Phys. Rev. Lett. 111, 089701 (2013); E. Muñoz, C.
J. Bolech, and S. Kirchner, Phys. Rev. Lett. 111, 089702 (2013).

53 Scaling of conductance through quantum dots with magnetic field, I. J. Hamad, C. Gazza, J. A. Andrade, A. A. Aligia, P. S. Cornaglia, and P. Roura-Bas, Phys. Rev. B 92, 195113 (2015); references therein.

54 Leading temperature dependence of the conductance in Kondo-correlated quantum dots. A. A. Aligia, J. Phys. Condens. Matter 30, 155304 (2018); references therein.

55 Scaling of the Kondo zero-bias peak in a hole quantum dot at finite temperatures, O. Klochan, A. P. Micolich, A. R. Hamilton, D. Reuter, A. D. Wieck, F. Reininghaus, M. Pletyukhov, and H. Schoeller, Phys. Rev. B 87, 201104(R) (2013).

56 Impact of capacitance and tunneling asymmetries on Coulomb blockade edges and Kondo peaks in nonequilibrium transport through molecular quantum dots, A. A. Aligia, P. Roura-Bas, and S. Florens, Phys. Rev. B 92, 035404 (2015).

57 From tunneling to contact in a magnetic atom: The nonequilibrium Kondo effect, D.-J. Choi, P. Abufager, L. Limot, and N. Lorente, J. Phys. Chem. 146, 092309 (2017).

58 Enhancing of nonlinear thermoelectric response of a correlated quantum dot in the Kondo regime by asymmetrically coupling to the leads, D. Pérez Daroca, P. Roura-Bas, and A. A. Aligia, Phys. Rev. B 97, 165433 (2018).

59 Kondo Resonance of a Co Atom Exchange Coupled to a Ferromagnetic Tip, D.-J. Choi, S. Guissart, M. Ormaza, N. Bachellier, O. Bengone, P. Simon, and L. Limot, et al. Nano Lett. 16, 6298 (2016).

60 Conductance-Driven Change of the Kondo Effect in a Single Cobalt Atom, D.-J. Choi, M. V. Rastei, P. Simon, and L. Limot, Phys. Rev. Lett. 108, 266803 (2012).

61 Conductance and Kondo Effect in a Controlled SingleAtom Contact, N. Néel, J. Kröger, L. Limot, K. Palotas, W. A. Hofer, and R. Berndt, Phys. Rev. Lett. 98, 016801 (2007).

62 Kondo effect of a Co atom on Cu(111) in contact with an iron tip, N. Néel, J. Kröger, and R. Berndt, Phys. Rev. B 82, 233401 (2010).

63 Spatially Extended Kondo State in Magnetic Molecules Induced by Interfacial Charge Transfer, U. G. E. Perera, H. J. Kulik, V. Iancu, L. G. G. V. Dias da Silva, S. E. Ulloa, N. Marzari, and S. W. Hla, Phys. Rev. Lett.105, 106601 (2010).

64 Observing the scattering phase shift of isolated Kondo impurities at surfaces, M. A. Schneider, L. Vitali, N. Knorr, and K. Kern, Phys. Rev. B 65, 121406(R) (2002).

65 Spin Control Induced by Molecular Charging in a Transport Junction, S. Karan, C, García, M- Karolak, D. Jacob, N. Lorente, and R. Berndt, Nano Lett. 18, 88 (2018).

66 Spin Polarization of the Split Kondo State, K. von Bergmann, M. Ternes, S. Loth, C. P. Lutz, and A. J. Heinrich Phys. Rev. Lett. 114, 076601 (2015).

67 Shifting the Voltage Drop in Electron Transport Through a Single Molecule, S. Karan, D. Jacob, M. Karolak, C. Hamann, Y. Wang, A. Weismann, A. I. Lichtenstein, and R. Berndt, Phys. Rev. Lett. 115, 016802 (2015).

68 Spin Manipulation by Creation of Single-Molecule Radical Cations, S. Karan, N. Li, Y. Zhang, Y. He, I-Po. Hong, H. Song, J-T. Lü, Y. Wang, L. Peng, K. Wu, G. S. Michelitsch, R. J. Maurer, K. Diller, K. Reuter, A. Weismann, and R. Berndt, Phys. Rev. Lett. 116, 027201 (2016).

69 Quantifying the leading role of the surface state in 
the Kondo effect of Co/Ag(111), M. Moro-Lagares, J. Fernández, P. Roura-Bas, M. R. Ibarra, A. A. Aligia, and D. Serrate, Phys. Rev. B 97, 235442 (2018).

70 Landauer formula for the current through an interacting electron region Y. Meir and N. S. Wingreen, Phys. Rev. Lett. 68, 2512 (1992).

71 Anderson model out of equilibrium: Noncrossingapproximation approach to transport through a quantum dot, N. S. Wingreen and Y. Meir, Phys. Rev. B 49, 11040 (1994).

72 Nonequilibrium self-energies, $\mathrm{Ng}$ approach, and heat current of a nanodevice for small bias voltage and temperature, A. A. Aligia, Phys. Rev. B 89, 125405 (2014); references therein.

73 Nonequilibrium transport through magnetic vibrating molecules, Phys. Rev. B 87, 195136 (2013) P. Roura-Bas, L. Tosi and A. A. Aligia; references therein.

${ }^{74}$ Non-equilibrium conductance through a benzene molecule in the Kondo regime, L. Tosi, P. Roura-Bas, and A. A. Aligia, J. Phys. Condens. Matter 24, 365301 (2012)

75 Nonequilibrium transport through a singlet-triplet Anderson impurity, P. Roura-Bas, A. A. Aligia Phys. Rev. B 80, 035308 (2009).

76 Nonequilibrium dynamics of a singlet-triplet Anderson impurity near the quantum phase transition, P. Roura-Bas, and A. A. Aligia, J. Phys. Condens. Matter. 22, 025602 (2010).

77 Non-equilibrium differential conductance through a quantum dot in a magnetic field, A. C. Hewson, J. Bauer, and A. Oguri, J. Phys.: Condens. Matter 17, 5413 (2005), and references therein.

78 Higher-order Fermi-liquid corrections for an Anderson impurity away from half-filling III: non-equilibrium transport. A. Oguri and A. C. Hewson, Phys. Rev. B 97, 035435 (2018); references therein.

79 At which magnetic field, exactly, does the Kondo resonance begin to split? A Fermi liquid description of the low-energy properties of the Anderson model, M. Filippone, C. P. Moca, A. Weichselbaum, J. von Delft, and C. Mora, Phys. Rev. B 98, 075404 (2018); references therein.

80 Fate of the spin-1/2 Kondo effect in the presence of temperature gradients, M. A. Sierra, R. López, and D. Sánchez, Phys. Rev. B 96, 085416 (2017).

${ }^{81}$ A thermally driven out-of-equilibrium two-impurity Kondo system, M. A. Sierra, R. López, and J. S. Lim, Phys. Rev. Lett. 121, 096801 (2018).

82 Anderson model out of equilibrium: Decoherence effects in transport through a quantum dot, R. Van Roermund, S. Y. Shiau, and M. Lavagna, Phys. Rev. B 81, 165115 (2010).

${ }^{83}$ Effective treatment of charge and spin fluctuations in dynamical and static atom-surface interactions, M. A. Romero, F. Flores, and E. C. Goldberg, Phys. Rev. B 80, 235427 (2009).

84 Corrected Kondo temperature beyond the conventional Kondo scaling limit, Z. Li, J. Wei, X. Zheng, Y. Yan, and H-G. Luo, J. Phys. Condens. Matter 29, 175601 (2017).

85 Solving Quantum Impurity Problems in and out of Equilibrium with the Variational Approach, Y. Ashida, T. Shi, M. C. Bañuls, J. I. Cirac, and E. Demler. Phys. Rev. Lett. 121, 026805 (2018).

86 The values of the renormalized parameters given in Ref. 53 have a mistake in one entry and in the values of $\Delta_{\rho}$ (not used). The correct table is presented in Ref. 54 .

87 Photoemission spectroscopy for the spin-degenerate Anderson model, H. O. Frota and L. N. Oliveira, Phys. Rev. B 33, 7871 (1986).

88 Width of the charge-transfer peak in the $S U(N)$ impurity Anderson model and its relevance to nonequilibrium transport, J. Fernández, F. Lisandrini, P. Roura-Bas, C. Gazza, and A. A. Aligia, Phys. Rev. B 97, 045144 (2018).

89 M. Moro-Lagares, Ph. D. thesis Engineering Spin Structures at the Atomic Scale (Prensas de la Universidad de Zaragoza, Zaragoza, Spain, 2017). ISBN 978-84-16935-833.

90 The NRG results of Fig. 7 have beeen calculated by G. Blesio using the NRG Ljubljana - open source numerical renormalization group code developed by R. Žitko, http://nrgljubljana.ijs.si (2014).

91 Spectral Properties of the Anderson Impurity Model, Comparison of Numerical Renormalization Group and NonCrossing Approximation, T. A. Costi, J. Kroha, P. Wolfle, Phys. Rev. B 53, 1850 (1996).

92 Thermoelectric Characterization of the Kondo Resonance in Nanowire Quantum Dots, A. Svilans, M. Josefsson, A. M. Burke, S. Fahlvik, C. Thelander, H. Linke, and M. Leijnse. Phys. Rev. Lett. 121, 206801 (2018).

${ }^{93}$ Kondo temperature when the Fermi level is near a step in the conduction density of states, J. Fernández, A. A. Aligia, P. Roura-Bas, and J. A. Andrade, Phys. Rev. B 95, 045143 (2017).

94 Conductance through $3 d$ impurities in gold chains doped with oxygen, M. A. Barral, S. Di Napoli, G. Blesio, P. Roura-Bas, A. Camjayi, L. O. Manuel, and A. A. Aligia; J. Chem. Phys. 146, 092315 (2017). 